\title{
Article \\ A Perturbation Approach for Lateral Excited Vibrations of a Beam-like Viscoelastic Microstructure Using the Nonlocal Theory
}

\author{
Cheng $\mathrm{Li}^{1}{ }^{1}$, Chengxiu Zhu ${ }^{1}$, Suihan $\mathrm{Sui}^{2}$ and Jianwei Yan ${ }^{3, *}$ \\ 1 School of Rail Transportation, Soochow University, Suzhou 215131, China; licheng@suda.edu.cn (C.L.); \\ zchengxiu@163.com (C.Z.) \\ 2 School of Mechanical Engineering, Shangqiu Institute of Technology, Shangqiu 476000, China; \\ suisuihan@163.com \\ 3 Engineering Research \& Development Centre for Underground Technology of Jiangxi Province, \\ School of Civil Engineering and Architecture, East China Jiaotong University, Nanchang 330013, China \\ * Correspondence: jianwei@mail.ustc.edu.cn
}

check for updates

Citation: Li, C.; Zhu, C.; Sui, S.; Yan, J. A Perturbation Approach for Lateral Excited Vibrations of a Beam-like

Viscoelastic Microstructure Using the Nonlocal Theory. Appl. Sci. 2022, 12, 40. https://doi.org/10.3390/ app12010040

Academic Editor: Xiao-Dong Yang

Received: 24 November 2021

Accepted: 16 December 2021

Published: 21 December 2021

Publisher's Note: MDPI stays neutral with regard to jurisdictional claims in published maps and institutional affiliations.

Copyright: (C) 2021 by the authors. Licensee MDPI, Basel, Switzerland. This article is an open access article distributed under the terms and conditions of the Creative Commons Attribution (CC BY) license (https:// creativecommons.org/licenses/by/ $4.0 /)$.

\begin{abstract}
In this paper, we investigate the lateral vibration of fully clamped beam-like microstructures subjected to an external transverse harmonic excitation. Eringen's nonlocal theory is applied, and the viscoelasticity of materials is considered. Hence, the small-scale effect and viscoelastic properties are adopted in the higher-order mathematical model. The classical stress and classical bending moments in mechanics of materials are unavailable when modeling a microstructure, and, accordingly, they are substituted for the corresponding effective nonlocal quantities proposed in the nonlocal stress theory. Owing to an axial elongation, the nonlinear partial differential equation that governs the lateral motion of beam-like viscoelastic microstructures is derived using a geometric, kinematical, and dynamic analysis. In the next step, the ordinary differential equations are obtained, and the time-dependent lateral displacement is determined via a perturbation method. The effects of external excitation amplitude on excited vibration are presented, and the relations between the nonlocal parameter, viscoelastic damping, detuning parameter, and the forced amplitude are discussed. Some dynamic phenomena in the excited vibration are revealed, and these have reference significance to the dynamic design and optimization of beam-like viscoelastic microstructures.
\end{abstract}

Keywords: microstructure; perturbation; nonlocal; viscoelastic; excited vibration

\section{Introduction}

Microscaled materials and microscaled structures have attracted considerable interest as sensors, resonators, vibrators, and attenuators [1-3]. Studies on small materials and structures with vibrations are indispensable in the current micro/nano-technology [4], and they are among the challenging tasks which micro-mechanical engineers have to face. The lateral vibration dominates the beam-like microstructures with an oscillation, of which some can be modeled as the lateral excited vibrations of fully clamped microbeams according to the external excitation and boundary constraints. In fact, the microstructures are often subjected to time-dependent external excitation, and, also, the fully clamped boundary is one of the most common end restrictions, both of which are easily seen in micro-electromechanical systems (MEMS) and other devices at the microscale [5]. However, in recent years, much attention has been paid to the free vibration of microscaled materials and structures [6-14]. For example, Lim et al. [6] investigated the free vibration of circular nanotubes subjected to both the torsional deformation and axial motion. The nonlinear history of strains is considered, and an infinite-order differential equation of motion is obtained via a variational principle. Li et al. [7] established a functionally graded Timoshenko beam model to reveal the size-dependent properties, including the strain gradient and nonlocal scale effects in its free vibration. Chwal and Muc [9] examined the free vibration of 
rectangular nanoplates using a refined shear deformation theory. Both the nonlocal strain and nonlocal stress were considered, and the Rayleigh-Ritz method was employed to solve the governing equations. The nonlocal strain and stress approaches were compared, and the effects of various parameters were demonstrated. Tang and Qing [13] presented the elastic buckling and free vibration of functionally graded microbeams via the stress-strain integral-differential equations that were solved by a Laplace transform technique.

As aforementioned, a large amount of studies regarding the free vibrational behaviors of beam-like microstructures can be found, but the excited vibration analyses of beam-like microstructures have not been investigated fully, and this is due to the lack of an excited vibration model containing intrinsic scale effects. Nevertheless, excited vibration is the main form of structural vibration in practical engineering [15]. With respect to a beam-like microstructure, it heavily depends on whether or not the intrinsic characteristic effects are taken into account, because the characteristic effects, such as the scale effect, cause changes in its lateral vibration. The process of discovering how to characterize the scale effect in microstructures is an important problem in micro-mechanics [16]. To this end, some modified non-classical continuum theories have been developed, of which the nonlocal theory is the most widely used size-dependent theory [17]. The nonlocal theory was first proposed by Eringen et al. $[18,19]$ to solve the stress distribution at a crack tip and wave propagation problems. It assumes that the stress at a point in the continuum is a function of strains at all points, and that is different from the classical continuum theories, because the latter considers the stress at a point as a function of the strain at only that point. The nonlocal theory belongs to a category with long-range interaction between atoms, but the classical continuum theories are just the opposite.

During the past several decades, the nonlocal theory has been applied extensively in micro/nano-mechanics [20-30]. Heireche et al. [21] examined the vibration characteristics in wave propagation of a pre-stressed double-walled carbon nanotube that was modeled as a Euler-Bernoulli beam model. The nonlocal theory was employed, and the van der Waals force was taken into consideration. Lim [22] proposed a new nonlocal stress theory to reveal the exact nanoscale effect in the equilibrium, governing equation, and static deflection of micro/nano-structures, in which a new physical quantity in continuum mechanics named the effective nonlocality is defined. Li [23] developed an analytical method to study the torsion of cylindrical micro/nano-structures where the effective nonlocal effect was involved, and the structural hardening effect was demonstrated. Behera and Chakraverty [25] reviewed recent advances on the nonlocal theory and its application in the vibration behaviors of carbon nanotubes using various microbeam models. They summarized the research status of the nonlocal theory relating to different types of complicating effects, nonlinearity, functionally graded material, and beam theories. Liu et al. [26] presented a piezoelectric nanoplate model using the nonlocal theory and Mindlin plate model, and, subsequently, investigated the nonlinear vibration to show the von Karman nonlinearity and micro-scale effect. Using the third-order shear deformation theory, Arefi and Rabczuk [27] presented a higher-order electro-elastic bending analysis of a piezoelectric doubly curved nanoshell resting on the Winkler-Pasternak foundation through the nonlocal theory. The nonlocal electro-elastic relations were introduced, and effects of external forces and voltages were discussed in detail. $\mathrm{Wu}$ and $\mathrm{Hu}$ [30] presented an overview of dynamic analyses of two-dimensional micro-structures, such as graphene sheets, using different nonlocal plate theories. The success of the theory is attributed to the characteristic scale parameters of materials introduced in the theoretical constitutive relation, making the theory suitable for predicting mechanical behaviors at micro-scale [31].

On the other hand, most previous studies only focused on the mechanical properties of elastic microstructures $[4,6-14,20-30]$. However, from a practical engineering standpoint, completely elastic structures are rare, and most of them should be modeled as viscoelastic structures [32-34]. Therefore, the investigation of excited vibrations of viscoelastic microbeams, which is closer to engineering application, is of concern. It is expected to be 
useful for understanding the special effects in the excited vibration of one-dimensional microscaled engineering structures.

In the present work, a fully clamped beam-like microstructure is described, and, subsequently, the lateral excited vibration is examined using the nonlocal theory and a perturbation method. The significant nonlocal effect in the lateral excitation is considered and the viscoelastic damping is also taken into account. The geometric nonlinearity resulting from the axial elongation is included in the problem model, which results in a nonlinear partial differential governing equation. For such a nonlinear equation, the perturbation methods, such as the method of multiple scales, are suitable. Hence, the method of multiple scales is applied into the governing equation directly in order to determine the amplitude and lateral displacement. Accordingly, it reveals some phenomena consistent with the prediction of classical vibration theory, as well as the unique mechanical behaviors only observed at a microscale. The present research provides a theoretical basis for promoting the application of micro-technology in the dynamics and control of viscoelastic beam-like microstructures.

\section{Theoretical Modeling and Formulations}

Considering a Euler-Bernoulli microbeam clamped at both ends with the length $\mathrm{L}$, density $\rho$, uniform cross sectional area A, we investigate its excited vibration that usually appears in the MEMS. The $\mathrm{X}$-axis is measured from the left side along the axial direction, while the $\mathrm{Y}$-axis is measured from the bottom along the transverse direction, shown in Figure 1. The dynamic equilibrium chart for a microbeam element straying from the left side $X$, with length $d X$ of the microbeam is illustrated in Figure 2, where $p(T) \cos (\pi X / L)$ is the external harmonic excitation at the time $\mathrm{T}$ and position $\mathrm{X}$ of unit length [35], $\mathrm{M}$ is the bending moment, $\mathrm{N}$ is the internal axial tension, $\mathrm{Q}$ is the shear force, and $\theta$ is the rotation angle with respect to $\mathrm{X}$-axis of the microbeam.

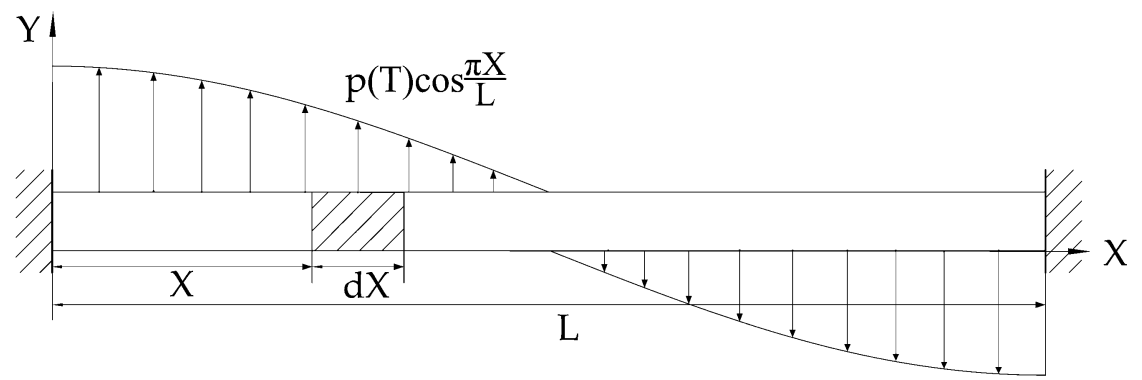

Figure 1. Sketch of the microbeam.

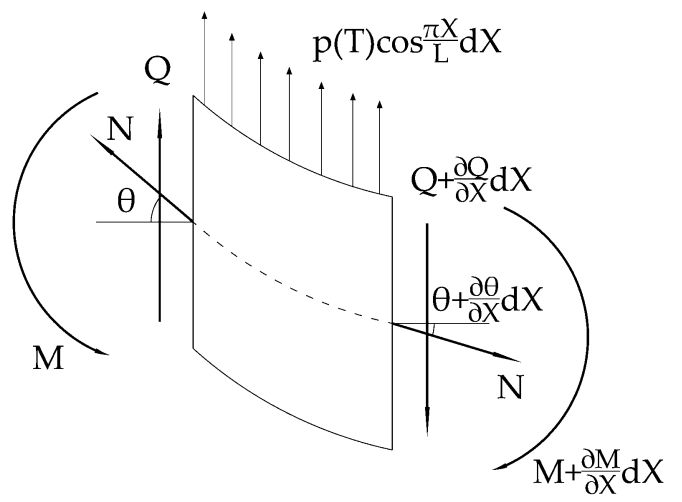

Figure 2. Dynamic equilibrium chart for a microbeam element. 
It is noticed that the shear deformation is ignored, according to the Euler-Bernoulli beam theory. Based on the d'Alembert principle, the equilibrium equation of the element provided with respect to the Y-axis can be obtained as

$$
\begin{aligned}
& Q-\left(Q+\frac{\partial Q}{\partial X} d X\right)+N \theta-N\left(\theta+\frac{\partial \theta}{\partial X} d X\right)+ \\
& p(T) \cos \frac{\pi X}{L} d X-\rho A \frac{\partial^{2} U}{\partial T^{2}} d X-\mu \frac{\partial U}{\partial T} d X=0
\end{aligned}
$$

where $U$ is the lateral displacement, the viscoelastic damping of the microstructures is considered, and $\mu$ is the viscoelastic damping coefficient.

Based on the moment balance condition and the relation between displacement and rotation angle, the above equation can be changed into the following one

$$
\frac{\partial^{2} M_{e f}}{\partial X^{2}}-N \frac{\partial^{2} U}{\partial X^{2}}+p(T) \cos \frac{\pi X}{L}-\rho A \frac{\partial^{2} U}{\partial T^{2}}-\mu \frac{\partial U}{\partial T}=0
$$

where $\mathrm{M}_{\mathrm{ef}}$ is the effective nonlocal bending moment defined in the nonlocal stress theory [22]. The axial tension $\mathrm{N}$ shown in Equation (2) caused by the lateral deformation is given by

$$
\frac{\mathrm{N}}{\mathrm{A}}=\mathrm{E} \frac{\int_{0}^{\mathrm{L}} \delta_{\mathrm{dX}} \mathrm{dX}}{\mathrm{L}}=\frac{\mathrm{E}}{\mathrm{L}} \int_{0}^{\mathrm{L}}\left[\sqrt{1+\left(\frac{\partial \mathrm{U}}{\partial \mathrm{X}}\right)^{2}}-1\right] \mathrm{dX}
$$

where $\delta_{\mathrm{dx}}$ is the axial elongation of a unit length $\mathrm{dX}$, and $\mathrm{E}$ is the Young's modulus of the microbeam.

Making Taylor expansion series for Equation (3) and dropping the higher-order terms yield

$$
\mathrm{N}=\frac{\mathrm{EA}}{2 \mathrm{~L}} \int_{0}^{\mathrm{L}}\left(\frac{\partial \mathrm{U}}{\partial \mathrm{X}}\right)^{2} \mathrm{dX}
$$

Substituting Equation (4) into (2), one obtains

$$
\frac{\partial^{2} M_{e f}}{\partial X^{2}}-\left[\frac{E A}{2 L} \int_{0}^{L}\left(\frac{\partial U}{\partial X}\right)^{2} d X\right] \frac{\partial^{2} U}{\partial X^{2}}+p(T) \cos \frac{\pi X}{L}-\rho A \frac{\partial^{2} U}{\partial T^{2}}-\mu \frac{\partial U}{\partial T}=0
$$

It is known that the relationship between the effective nonlocal bending moment and lateral displacement based on the nonlocal stress theory is [22,23]

$$
M_{e f}=-E I \sum_{n=1}^{\infty}\left(e_{0} a\right)^{2(n-1)} \frac{\partial^{2 n} U}{\partial X^{2 n}}
$$

where $\mathrm{I}=\iint_{\mathrm{A}} \mathrm{Y}^{2} \mathrm{dA}$ is the usual area moment of inertia of the microstructural cross section, $\mathrm{a}$ is an internal characteristic length scale and $\mathrm{e}_{0}$ is a constant dependent on material properties [19]. The magnitude of $\mathrm{e}_{0}$ is determined experimentally or approximated by matching the theoretical predictions with those of molecular dynamics simulations. The combination of $\mathrm{e}_{0} \mathrm{a}$ is defined as a nonlocal modulus, which approaches the Dirac delta function in the limit that the nonlocal modulus is much smaller than the external characteristic length of the microbeam. In that case, the classical continuum mechanics will be recovered. Moreover, it is interesting that the effective nonlocal bending moment turns into the classical bending moment when $n=1$ in Equation (6). That is, the effective nonlocal bending moment is an infinite higher-order variable based on the classical counterpart. However, with the increase of $n$, the influence of higher-order terms will be smaller and smaller because $e_{0} a$ is a small quantity. 
Consequently, only the first two terms in the effective nonlocal bending moment are considered and further substitute it into Equation (5), one can obtain the following nonlinear partial differential equation that governs the lateral vibration as

$$
\begin{aligned}
& \mathrm{EI}\left[\frac{\partial^{4} \mathrm{U}}{\partial \mathrm{X}^{4}}+\left(\mathrm{e}_{0} \mathrm{a}\right)^{2} \frac{\partial^{6} \mathrm{U}}{\partial \mathrm{X}^{6}}\right]+\left[\frac{\mathrm{EA}}{2 \mathrm{~L}} \int_{0}^{\mathrm{L}}\left(\frac{\partial \mathrm{U}}{\partial \mathrm{X}}\right)^{2} \mathrm{dX}\right] \frac{\partial^{2} \mathrm{U}}{\partial \mathrm{X}^{2}}- \\
& \mathrm{p}(\mathrm{T}) \cos \frac{\pi \mathrm{X}}{\mathrm{L}}+\rho \mathrm{A} \frac{\partial^{2} \mathrm{U}}{\partial \mathrm{T}^{2}}+\mu \frac{\partial \mathrm{U}}{\partial \mathrm{T}}=0
\end{aligned}
$$

It is shown that, unlike the classical vibration equation of beams with a fourth-order differential equation [36], the governing equation based on the nonlocal theory is a higherorder one. For the above partial differential Equation (7), it is difficult to determine the exact solutions directly. In this paper, we attempt to observe effects of the nonlocal parameter on the amplitude-frequency characteristics of the microbeam, in an effort to separate the spatial and temporal variables. According to the form of the external force, the approximate solution for the lateral vibration of the microbeam can be assumed as

$$
\mathrm{U}(\mathrm{X}, \mathrm{T})=\mathrm{S}(\mathrm{T}) \cos \frac{\pi \mathrm{X}}{\mathrm{L}}
$$

where $S$ is the time-dependent displacement and $\cos (\pi \mathrm{X} / \mathrm{L})$ is the approximate resonance vibration form of the microbeam clamped at both ends.

By use of Equation (8), the governing Equation (7) then becomes

$$
\mathrm{EI}\left[\frac{\pi^{4}}{\mathrm{~L}^{4}} \mathrm{~S}-\left(\mathrm{e}_{0} \mathrm{a}\right)^{2} \frac{\pi^{6}}{\mathrm{~L}^{6}} \mathrm{~S}\right]-\frac{\mathrm{EA} \pi^{4}}{4 \mathrm{~L}^{4}} \mathrm{~S}^{3}-\mathrm{p}(\mathrm{T})+\rho \mathrm{A} \frac{\mathrm{d}^{2} \mathrm{~S}}{\mathrm{dT}^{2}}+\mu \frac{\mathrm{d} S}{\mathrm{dT}}=0
$$

Introduce the following non-dimensional variables and parameters:

$$
\mathrm{s}=\mathrm{S} \sqrt{\frac{\mathrm{A}}{\mathrm{I}}}, \omega_{0}=\sqrt{\frac{\mathrm{EI} \pi^{4}}{\rho \mathrm{AL}^{4}}}, \mathrm{t}=\omega_{0} \mathrm{~T}, \varsigma=\frac{\mu}{\rho \mathrm{A}}, \tau=\frac{\mathrm{e}_{0} \mathrm{a}}{\mathrm{L}}, \varepsilon=\frac{\omega_{0}^{2}}{4}
$$

where $\varepsilon$ is a small variable and $\tau$ is the non-dimensional nonlocal parameter which describes the nanoscale effects. For a microbeam with certain material and length, the nondimensional nonlocal parameter is a constant. Using the above variables and parameters, Equation (9) can be cast into the dimensionless form as follows

$$
\omega_{0}^{2} \frac{d^{2} s}{d t^{2}}-\varepsilon s^{3}+\omega_{0}^{2} s-\omega_{0}^{2} \pi^{2} \tau^{2} s+\varsigma \omega_{0} \frac{d s}{d t}=\frac{A_{m} \sin (\Omega T)}{\rho \sqrt{A I}}
$$

where $\mathrm{p}(\mathrm{T})=\mathrm{A}_{\mathrm{m}} \sin (\Omega \mathrm{T})$ is assumed and $\mathrm{A}_{\mathrm{m}}$ is the amplitude of the external excitation, $\Omega$ is the excitation frequency. Further, the following equation can be determined and shown as

$$
\omega_{0}^{2} \frac{d^{2} s}{d t^{2}}-\varepsilon s^{3}+\omega_{0}^{2} s-\omega_{0}^{2} \pi^{2} \tau^{2} s+\varepsilon \kappa \frac{d s}{d t}=\varepsilon a_{m} \sin (\omega t)
$$

where $\mathrm{a}_{\mathrm{m}}=\frac{\mathrm{A}_{\mathrm{m}}}{\rho \varepsilon \sqrt{\mathrm{AI}}}, \omega=\frac{\Omega}{\omega_{0}} \approx 1$ and $\kappa=\frac{\zeta \omega_{0}}{\varepsilon}$.

\section{Perturbation Analyses}

For a weak nonlinear system, the perturbation methods, such as the method of multiple scales, are valid [37,38], and these can transform the nonlinear equation into a set of linear ordinary differential equations. Considering the method of multiple scales, and, assuming that $\mathrm{T}_{\mathrm{n}}=\varepsilon^{\mathrm{n}} \mathrm{t}$, one obtains

$$
\frac{\mathrm{d}}{\mathrm{dt}}=\frac{\partial}{\partial \mathrm{T}_{0}}+\varepsilon \frac{\partial}{\partial \mathrm{T}_{1}}+\cdots=\mathrm{D}_{0}+\varepsilon \mathrm{D}_{1}+\cdots
$$




$$
\frac{\mathrm{d}^{2}}{\mathrm{dt}^{2}}=\frac{\mathrm{d}}{\mathrm{dt}}\left(\frac{\partial}{\partial \mathrm{T}_{0}}+\varepsilon \frac{\partial}{\partial \mathrm{T}_{1}}+\cdots\right)=\mathrm{D}_{0}^{2}+2 \varepsilon \mathrm{D}_{0} \mathrm{D}_{1}+\cdots
$$

In the present work, we only adopt the first-order approximation, which is sought in the form

$$
\mathrm{s}(\mathrm{t} ; \varepsilon)=\mathrm{s}_{0}\left(\mathrm{~T}_{0}, \mathrm{~T}_{1}\right)+\varepsilon \mathrm{s}_{1}\left(\mathrm{~T}_{0}, \mathrm{~T}_{1}\right)+\cdots,
$$

where $T_{0}$ is a fast time scale characterizing motion occurring at the natural frequencies of the corresponding linear vibration system, $\mathrm{T}_{1}$ is a slow time scale characterizing the modulation of the amplitudes and phases.

The combination of Equations (12)-(15) yields

$$
\begin{aligned}
& \omega_{0}^{2}\left(\mathrm{D}_{0}^{2}+2 \varepsilon \mathrm{D}_{0} \mathrm{D}_{1}\right)\left(\mathrm{s}_{0}+\varepsilon \mathrm{s}_{1}\right)-\varepsilon\left(\mathrm{s}_{0}+\varepsilon \mathrm{s}_{1}\right)^{3}+\omega_{0}^{2}\left(\mathrm{~s}_{0}+\varepsilon \mathrm{s}_{1}\right) \\
& -\omega_{0}^{2} \pi^{2} \tau^{2}\left(\mathrm{~s}_{0}+\varepsilon \mathrm{s}_{1}\right)+\varepsilon \kappa\left(\mathrm{D}_{0}+\varepsilon \mathrm{D}_{1}\right)\left(\mathrm{s}_{0}+\varepsilon \mathrm{s}_{1}\right)=\varepsilon \mathrm{a}_{\mathrm{m}} \sin \left(\omega \mathrm{T}_{0}\right)
\end{aligned}
$$

Considering the excitation is $O(\varepsilon)$, we assume $\omega-\omega_{0}$ is $O(\varepsilon)$ for consistency. Hence, we introduce a relation $\omega=\omega_{0}+\varepsilon v$, where $v$ is a detuning parameter (i.e., the frequency of disturbing force, $v=\mathrm{O}(1)$ ) that is a notation for the mathematical solution of a nonlinear vibration with cubic nonlinearity [38]. Equating the coefficients of $\varepsilon^{n}$ with $n=0$ and 1 on both sides in the expansion of Equation (16) yields

$$
\begin{gathered}
\omega_{0}^{2} \mathrm{D}_{0}^{2} \mathrm{~s}_{0}+\left(\omega_{0}^{2}-\omega_{0}^{2} \pi^{2} \tau^{2}\right) \mathrm{s}_{0}=0 \\
\omega_{0}^{2} \mathrm{D}_{0}^{2} \mathrm{~s}_{1}+\left(\omega_{0}^{2}-\omega_{0}^{2} \pi^{2} \tau^{2}\right) \mathrm{s}_{1}=\mathrm{s}_{0}^{3}-2 \omega_{0}^{2} \mathrm{D}_{0} \mathrm{D}_{1} \mathrm{~s}_{0}-\mathrm{kD}_{0} \mathrm{~s}_{0}+\mathrm{a}_{\mathrm{m}} \sin \left(\omega_{0} \mathrm{~T}_{0}+v \mathrm{~T}_{1}\right)
\end{gathered}
$$

From Equation (17) we obtain

$$
\mathrm{s}_{0}=\mathrm{B}\left(\mathrm{T}_{1}\right) \mathrm{e}^{\mathrm{i} \sqrt{1-\pi^{2} \tau^{2}} \mathrm{~T}_{0}}+\mathrm{cc}
$$

where $\mathrm{B}$ is a complex function with respect to $\mathrm{T}_{1}$ and $\mathrm{cc}$ is the conjugate complex number of its left terms, similarly hereinafter. It can be determined that $c c=\bar{B}\left(T_{1}\right) e^{-i \sqrt{1-\pi^{2} \tau^{2}} T_{0}}$ in Equation (19), and $\bar{B}$ is the conjugate function of $B$.

Substituting Equation (19) into (18) yields

$$
\begin{aligned}
& \omega_{0}^{2} \mathrm{D}_{0}^{2} \mathrm{~s}_{1}+\left(\omega_{0}^{2}-\omega_{0}^{2} \pi^{2} \tau^{2}\right) \mathrm{s}_{1}=\mathrm{B}^{3} \mathrm{e}^{3 \mathrm{i} \sqrt{1-\pi^{2} \tau^{2}} \mathrm{~T}_{0}}+ \\
& \left(3 \mathrm{~B}^{2} \overline{\mathrm{B}}-2 \mathrm{i} \omega_{0}^{2} \sqrt{1-\pi^{2} \tau^{2}} \mathrm{D}_{1} \mathrm{~B}-\mathrm{i} \kappa \sqrt{1-\pi^{2} \tau^{2}} \mathrm{~B}+\frac{1}{2 \mathrm{i}} \mathrm{a}_{\mathrm{m}} \mathrm{e}^{\mathrm{i} \mathrm{T}_{1} v}\right) \mathrm{e}^{\mathrm{i} \sqrt{1-\pi^{2} \tau^{2}} \mathrm{~T}_{0}}+\mathrm{cc}
\end{aligned}
$$

where a zero-order approximation $\omega_{0}=\sqrt{1-\pi^{2} \tau^{2}}$ is adopted from Equation (19). Additionally, secular terms in Equation (20) must vanish in order to obtain a periodic solution for $\mathrm{s}_{1}$. As a result, the following relation and solution can be derived by eliminating the terms which produce secular terms as

$$
\begin{gathered}
3 B^{2} \bar{B}-2 i \omega_{0}^{2} \sqrt{1-\pi^{2} \tau^{2}} D_{1} B-i \kappa \sqrt{1-\pi^{2} \tau^{2}} B+\frac{1}{2 i} a_{m} e^{i T_{1} v}=0 \\
s_{1}=-\frac{1}{8\left(1-\pi^{2} \tau^{2}\right) \omega_{0}^{2}} B^{3} e^{3 i \sqrt{1-\pi^{2} \tau^{2}}} T_{0}+c c
\end{gathered}
$$

Assuming $B=\frac{1}{2} b e^{i \beta}$, where $b$ is the amplitude of excited vibration and $\beta$ is a real function with respect to $T_{1}$ and substituting it into Equation (21) then separating the real-parts and imaginary-parts in the results yields

$$
\begin{aligned}
& \mathrm{b} \beta^{\prime}=-\frac{3}{8 \omega_{0}^{2} \sqrt{1-\pi^{2} \tau^{2}}} \mathrm{~b}^{3}-\frac{\mathrm{am}}{2 \omega_{0}^{2} \sqrt{1-\pi^{2} \tau^{2}}} \sin \left(v \mathrm{~T}_{1}-\beta\right) \\
& \mathrm{b}^{\prime}=-\frac{\mathrm{b} \kappa}{2 \omega_{0}^{2}}-\frac{\mathrm{a}_{\mathrm{m}}}{2 \omega_{0}^{2} \sqrt{1-\pi^{2} \tau^{2}}} \cos \left(v \mathrm{~T}_{1}-\beta\right)
\end{aligned}
$$


Introduce a variable $\gamma=v \mathrm{~T}_{1}-\beta$ and so $\gamma^{\prime}=v-\beta^{\prime}$, Equation (23) then becomes

$$
\begin{aligned}
& \mathrm{b} \gamma^{\prime}=\mathrm{bv}+\frac{3}{8 \omega_{0}^{2} \sqrt{1-\pi^{2} \tau^{2}}} \mathrm{~b}^{3}+\frac{\mathrm{a}_{\mathrm{m}}}{2 \omega_{0}^{2} \sqrt{1-\pi^{2} \tau^{2}}} \sin \gamma \\
& \mathrm{b}^{\prime}=-\frac{\mathrm{b} \kappa}{2 \omega_{0}^{2}}-\frac{\mathrm{a}_{\mathrm{m}}}{2 \omega_{0}^{2} \sqrt{1-\pi^{2} \tau^{2}}} \cos \gamma
\end{aligned}
$$

We seek a stable periodic solution in this study, so $\mathrm{b}$ and $\gamma$ should approach stability, hence

$$
\left(b v+\frac{3}{8 \omega_{0}^{2} \sqrt{1-\pi^{2} \tau^{2}}} b^{3}\right)^{2}+\left(\frac{b \kappa}{2 \omega_{0}^{2}}\right)^{2}=\left(\frac{a_{m}}{2 \omega_{0}^{2} \sqrt{1-\pi^{2} \tau^{2}}}\right)^{2}
$$

Equation (25) is the frequency response equation, and, in fact, it contains the implicit function relations among amplitude $b$, detuning parameter $v$ and amplitude of disturbing force $A_{m}$.

\section{Results and Discussion}

Figure 3 shows the relationship between the detuning parameter and the forced amplitude, in which $\mathrm{a}_{\mathrm{m}}=\omega_{0}=\mathrm{k}=0.1$ is assumed. Note that the condition of $\tau=0$ in Figure 3 means zero dimensionless nonlocal parameter. Considering that the dimensionless nonlocal parameter is defined as the ratio of internal and external characteristic scale parameters, $\tau=0$ indicates that the external scale parameter (e.g., length of a beam) is much larger than the internal scale parameter (e.g., a lattice parameter), or the internal characteristic scale can be ignored in comparison with the corresponding external counterpart. On this condition, the size of the beam deviates from the micro-scale, and, as a result, a classical macrobeam and related results of classical vibration theory recover. However, the condition of $\tau=0.15$ represents that the values of internal and external characteristic scale parameters are comparable, as, notably, the magnitudes of internal and external characteristic scales are almost at the same level, namely, the size of the beam is at a micro-scale. For a microbeam, the external characteristic scale parameter is also in the order of microns/nanometers, the nonlocal parameter that measures the significant nonlocal effect should not be zero; that is the essence of the nonlocal theory. It is found that, when the absolute value of the detuning parameter becomes larger, the forced amplitude becomes smaller. Moreover, it is noted that, in the ranges of $v_{1}-v_{2}$ and $v_{1}^{\prime}-v_{2}^{\prime}$, for the same detuning parameter, there are multiple stable-steady excited vibrations with different amplitudes. Therefore, a leap phenomenon exists in the two steady vibrations. It is observed that such a phenomenon also exists in the case of $\tau=0.15$, which indicates that some mechanisms at the micro-scale are identical to those at a macro-scale. For example, the leap phenomenon and related characteristics (e.g., a softening effect) exist in both the classical and nonlocal vibration models. However, some mechanisms are unique only at small-scales. Comparing the case of $\tau=0$ with $\tau=0.15$, that is, without and with the nonlocal effect, we find that the nonlocal effect deflects the curve in Figure 3 where the relationship between forced amplitude and detuning parameter changes. In the range of $v_{3}^{\prime}-v^{\prime}{ }_{4}$, the forced amplitude decreases when the nonlocal effect is taken into account, while beyond the range, the amplitude increases, which indicates that the nonlocal effect significantly affects the forced amplitude. Therefore, the nonlocal effect plays an indispensable role in the analysis of excited vibrations at the micro-scale.

Figure 4 shows the relationship between the forced amplitude and the viscoelastic damping coefficient, in which $\mathrm{a}_{\mathrm{m}}=\omega_{0}=0.1$ is assumed. The softening effect is observed again where the forced amplitude gradually decreases with the increase of the viscoelastic damping coefficient, and the decreasing speed is slow at first, and then fast, and finally the curves tend to be flat. In addition, it has been observed that the dimensionless viscoelastic damping parameter has a significant influence on the nonlocal effect in the forced amplitude corresponding to the same detuning parameter. For the case of $v=10$, the nonlocal effect increases the forced amplitude, and the difference becomes a little larger as $k$ increases and 
then gradually decreases when $\mathrm{k}$ is more than 0.6 . Corresponding to $v=-10$, the forced amplitude with a nonlocal effect $(\tau=0.15)$ is smaller than that without the nonlocal effect $(\tau=0)$ when $\kappa$ is small, but with the increase of $\kappa$, the situation reverses, namely, the forced amplitude with nonlocal effect exceeds that without the nonlocal effect. Consequently, there is a coupling between the effects of the nonlocal parameter, viscoelastic damping, and detuning parameter on the forced amplitude.

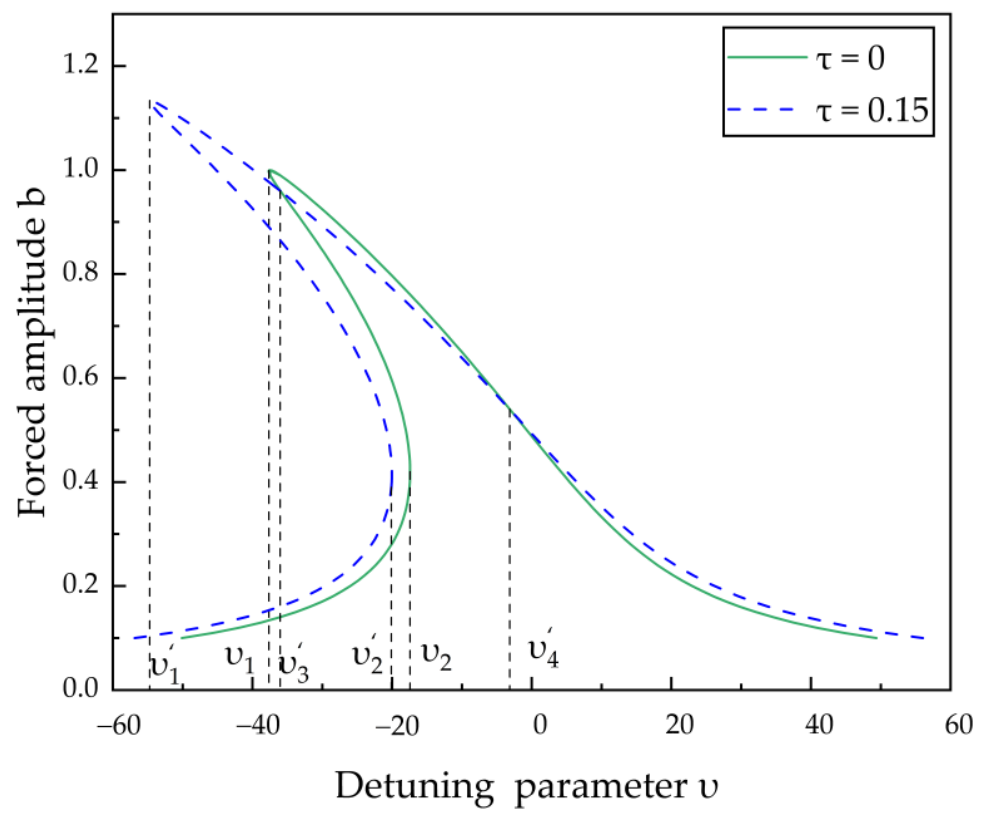

Figure 3. Frequency response curves of the micro-beam.

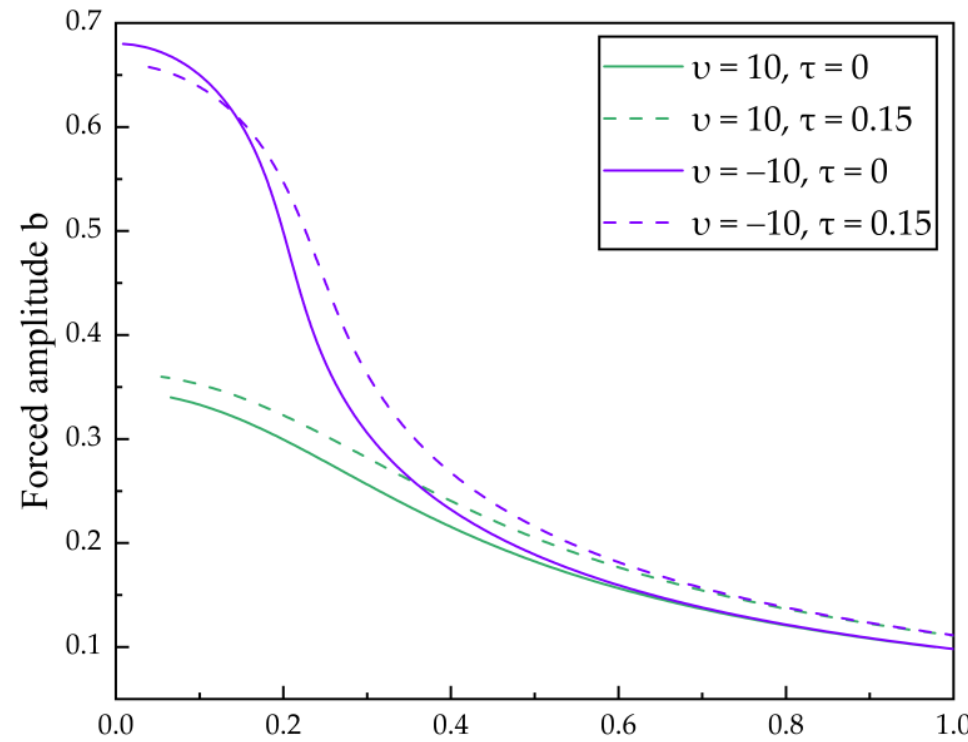

Dimensionless viscoelastic damping coefficient $\kappa$

Figure 4. Forced amplitude versus viscoelastic damping coefficient.

The expression of detuning parameter can be obtained from Equation (25), given by

$$
v= \pm \sqrt{\left(\frac{2 A_{m}}{\rho \sqrt{\mathrm{Al} b} \omega_{0}^{4} \sqrt{1-\pi^{2} \tau^{2}}}\right)^{2}-\left(\frac{\kappa}{2 \omega_{0}^{2}}\right)^{2}}-\frac{3 b^{2}}{8 \omega_{0}^{2} \sqrt{1-\pi^{2} \tau^{2}}} .
$$


In Equation (26), assuming $\kappa=\rho \sqrt{\mathrm{AI}}=\omega_{0}=0.1$, we obtain the relations between the excitation amplitude and forced amplitude for different detuning parameters and nonlocal parameters, shown in Figure 5. Unlike the softening effect shown before, a hardening effect is observed. The results show that for the case of $v=-20$ with a small excitation amplitude, there are multiple forced amplitudes corresponding to the same excitation amplitude, which is caused by the aforementioned leap phenomenon. On the other hand, while the excitation amplitude becomes larger, the forced amplitude increases with the increase in the excitation amplitude, but the rate of increase is smaller and smaller. In addition, the forced amplitude decreases with an increase in the detuning parameter. For the case of $v=-10$ and $v=-20$, the forced amplitude with a nonlocal effect $(\tau=0.15)$ is smaller than that without a nonlocal effect $(\tau=0)$ or the classical counterpart. For the case of $v=20$, the nonlocal result $(\tau=0.15)$ is larger than the classical result $(\tau=0)$. This is because the main vibration function is deflected as a result of the existence of the nonlocal effect. Predictably, the larger the nonlocal parameter is, the larger the numerical result is.

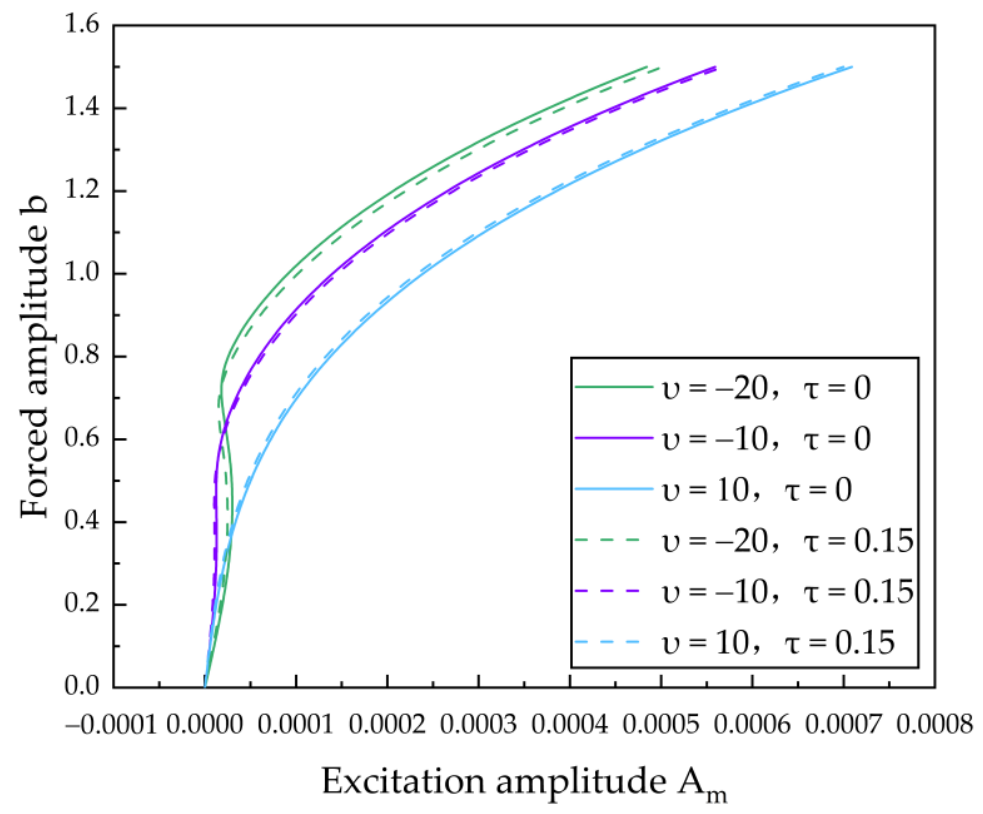

Figure 5. Forced amplitude versus excitation amplitude with different detuning parameters.

Assuming $\omega_{0}=0.1$ and $v=10$, Figure 6 shows the variation of forced amplitude with excitation amplitude under different dimensionless viscoelastic damping parameters and nonlocal parameters. In addition to a significant nonlocal effect, where the nonlocality reduces the forced amplitude with the same other conditions, it is also observed that, for different viscoelastic damping parameters, the forced amplitude increases with an increase in the excitation amplitude. Hence, the hardening phenomenon occurs again. Therefore, the present work proves that both the softening and hardening effects can be found in a micro/nano-structure or device, and this is similar to the nonlinear system with macrostructures. It is worth mentioning that, in early years, there was controversy about whether micro/nano-structures showed nonlinear softening or hardening mechanisms [22]. Li et al. [24] and Lim et al. [39] confirmed that both exist at the micro/nanoscale. The present results support this point again. In addition, the smaller the viscoelastic damping parameter, the larger the forced amplitude. For a completely elastic microbeam, the forced amplitude will reach the maximum. 


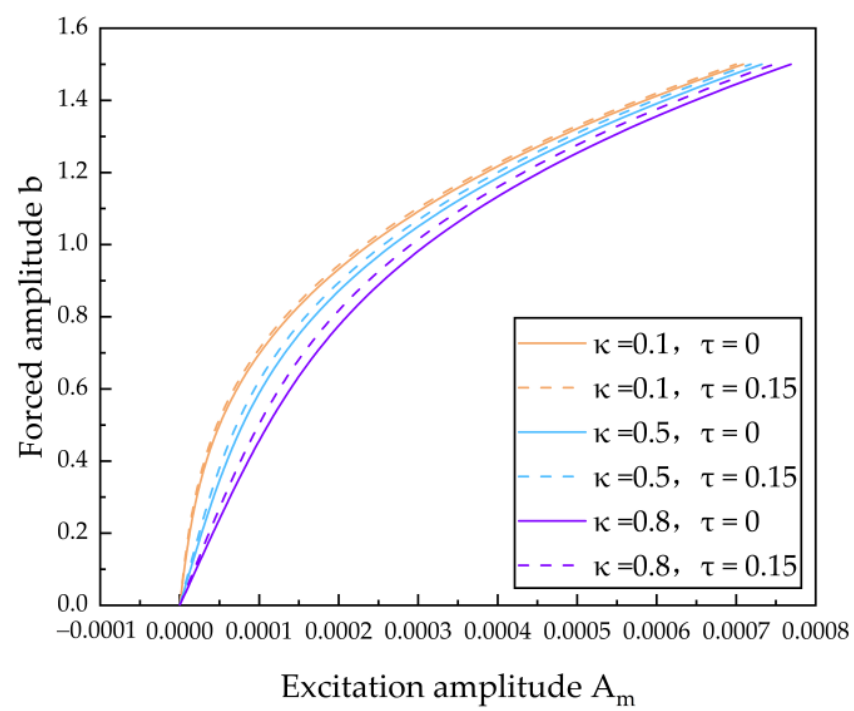

Figure 6. Forced amplitude versus excitation amplitude with a different viscoelastic damping coefficient.

Additionally, we gain the expression of complex function B as

$$
\mathrm{B}\left(\mathrm{T}_{1}\right)=\frac{1}{2} \mathrm{~b}_{0} \mathrm{e}^{\mathrm{i}\left[\left(\sqrt{\left(\frac{\mathrm{am}}{2 \mathrm{~b}_{0} \omega_{0}^{2} \sqrt{1-\pi^{2} \tau^{2}}}\right)^{2}-\left(\frac{\kappa}{2 \omega_{0}^{2}}\right)^{2}}-\frac{3 \mathrm{~b}_{0}^{2}}{8 \omega_{0}^{2} \sqrt{1-\pi^{2} \tau^{2}}}\right) \mathrm{T}_{1}+\beta_{0}\right]}
$$

where $b_{0}$ and $\beta_{0}$ are integral constants and they can be determined by certain initial conditions.

Using the combination of Equations (15), (19), (22) and (27), one obtains the nondimensional time-dependent displacement as

$$
\mathrm{s}=\frac{1}{2} \mathrm{~b}_{0} \mathrm{e}^{\mathrm{i} \Lambda}-\frac{\mathrm{b}_{0}^{3} \varepsilon}{64 \sqrt{1-\pi^{2} \tau^{2}} \omega_{0}^{2}} \mathrm{e}^{3 \mathrm{i} \Lambda}
$$

where $\Lambda=\left(\sqrt{\left(\frac{a_{m}}{2 b_{0} \omega_{0}^{2} \sqrt{1-\pi^{2} \tau^{2}}}\right)^{2}-\left(\frac{k}{2 \omega_{0}^{2}}\right)^{2}}-\frac{3 b_{0}^{2}}{8 \omega_{0}^{2} \sqrt{1-\pi^{2} \tau^{2}}}\right) \varepsilon t+\sqrt{1-\pi^{2} \tau^{2}} t+\beta_{0}$. Relations between the real-part of the time-dependent displacement and dimensionless time coordinate given in Equation (28) can also be illustrated in Figure 7, where $b_{0}=\beta_{0}=\varepsilon=1$, $\omega_{0}=\mathrm{k}=0.1$ and $\mathrm{a}_{\mathrm{m}}=0.1$ are assumed; it indicates that the nonlocal parameter $\tau$ causes the displacement to draft along the time coordinate axis. Consequently, the nonlocal hysteresis effect is found in the lateral excited vibration of a beam-like microstructure, which is mainly caused by the small-scale effect and viscoelastic effect. It is noted that the hysteresis effect has not been reported in the nonlocal free vibration of micro/nanostructures $[6-14,20-30]$. Resultingly, it is a special phenomenon in excited vibration. With a longer time, the nonlocal hysteresis effect seems more obvious.

From Equations (8) and (28) we can deduce the dimensionless lateral displacement as:

$$
\mathrm{u}(\mathrm{x}, \mathrm{t})=\left(\frac{1}{2} \mathrm{~b}_{0} \mathrm{e}^{\mathrm{i} \Lambda}-\frac{\mathrm{b}_{0}^{3} \varepsilon}{64 \sqrt{1-\pi^{2} \tau^{2}} \omega_{0}^{2}} \mathrm{e}^{3 \mathrm{i} \Lambda}\right) \cos (\pi \mathrm{x}) .
$$

where $\mathrm{u}=\mathrm{U} \sqrt{\frac{\mathrm{A}}{\mathrm{I}}}, \mathrm{x}=\frac{\mathrm{X}}{\mathrm{L}}$ and the cubic nonlinearity is reflected again here. The evolvement of lateral displacement with respect to axial and time coordinates is given in Figure 8, where $\tau=0.1$ is adopted besides the assumptions in Figure 7. It clearly reveals the changes of lateral excited displacement with time and spatial variables, which are different from the results in the classical beam excited vibration, because of the nonlocal effect. For example, the displacement of the left and right halves of the microbeam is not symmetrical. Addi- 
tionally, the peak values of the real-part displacement (or imaginary-part) are different at different moments. Since most of the numerical results in this paper are shown in dimensionless form, they cannot be directly compared with the classical excited vibration results. However, from the qualitative trend, many conclusions reported herein are consistent with the classical excited vibration, because of the existence of external harmonic excitation and internal viscoelasticity. Hence, the present numerical results can be verified qualitatively. In addition, some phenomena, unique to the excited vibration of microbeams, are found in the numerical results due to the existence of nonlocality.

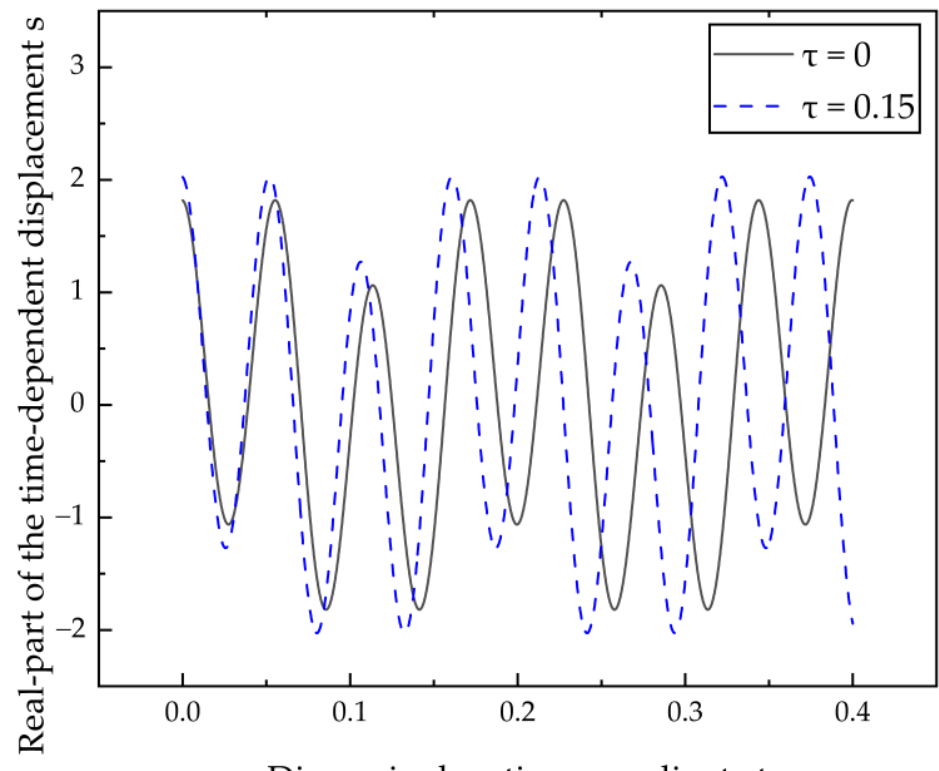

Dimensionless time coordinate $t$

Figure 7. Relations between the time-dependent displacement and the time coordinate.

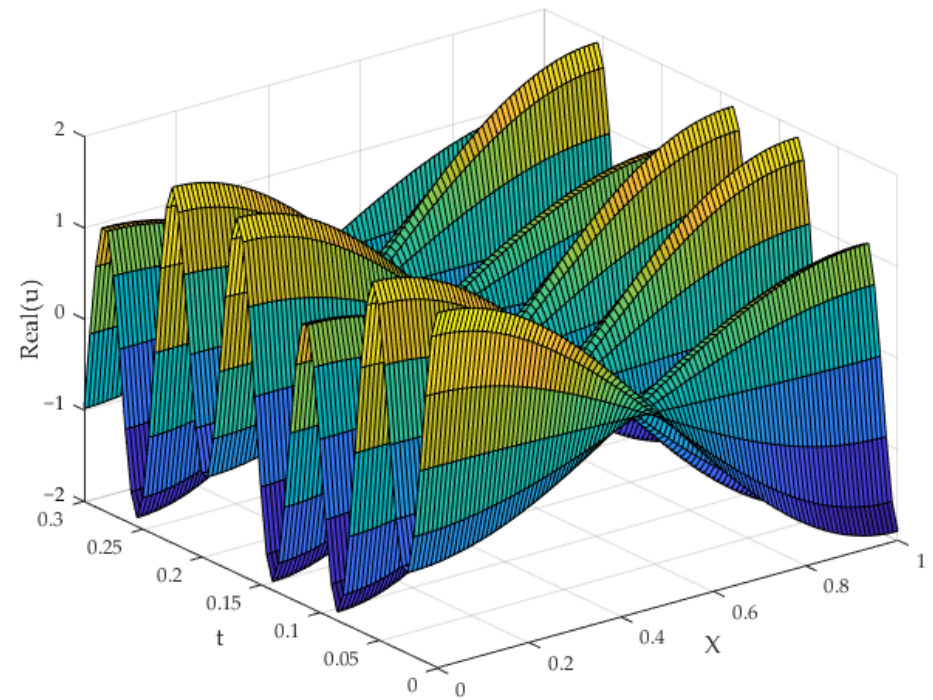

(a)

Figure 8. Cont. 


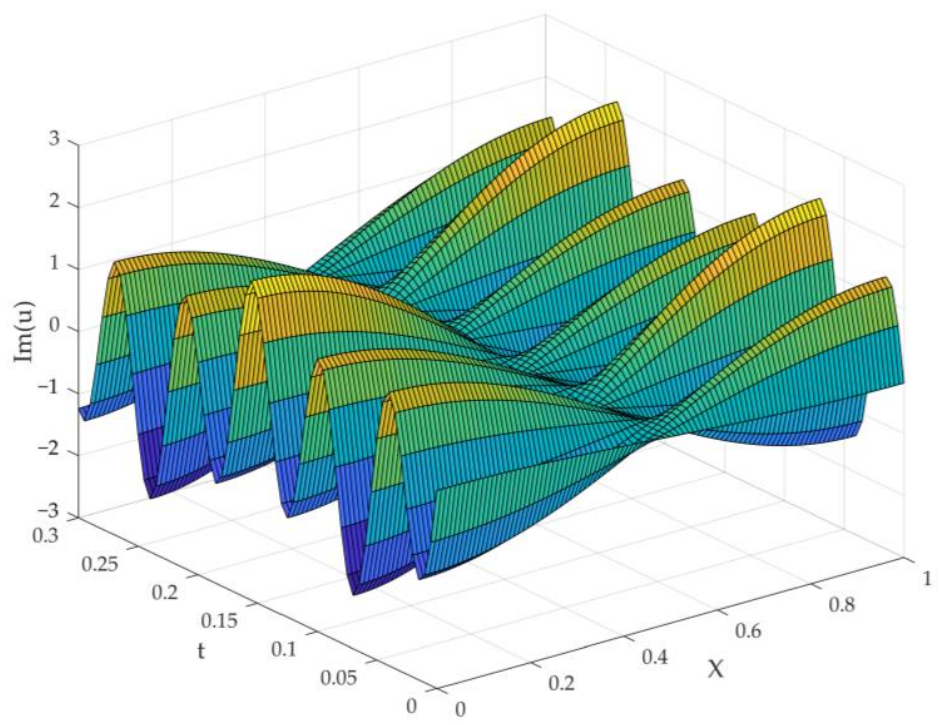

(b)

Figure 8. The evolvement of lateral displacement: (a) the real-part; (b) the imaginary-part.

Finally, further research into this topic can be undertaken: (i) The one-dimensional viscoelastic microbeam can be extended to microplate and microshell structures, and their lateral excited vibration responses need to be analyzed; (ii) beyond the nonlocal effect, the strain gradient effect, surface effect, and quantum effect of the excited vibration of viscoelastic microstructures can be considered, which are helpful to explain the key dynamics mechanisms at the microscale; (iii) based on the above two points, it is necessary to develop new perturbation methods in order to solve complex dynamic equations with higher dimensions and more variables.

\section{Conclusions}

The lateral excited vibration of a beam-like microstructure, resulting from timedependent excitation and an axial elongation, has been examined based on the nonlocal theory. The viscoelastic damping parameter was involved in modeling the excited vibration of microbeams when an external harmonic excitation is considered. The perturbation method was used while solving the nonlinear equation of motion, and the excited vibration characteristics were analyzed. Our priority revolved around revealing the nonlocal and viscoelastic damping effects in the lateral excited vibration. The following conclusions were obtained:

1. The nonlocal effect deflects the main vibration function and significantly affects the magnitude of the forced amplitude. There is a leap phenomenon that is similar to the classical vibration theory. The nonlocal parameter causes the displacement to draft along the time coordinate, and, hence, the hysteresis effect in nonlocal excited vibration is revealed.

2. The forced amplitude decreases with the increase of the viscoelastic damping, and the variation of the viscoelastic damping has a significant influence on the nonlocal effect in the forced amplitude. The nonlocal effects on the forced amplitude are different with different detuning parameters, because of the leap phenomenon. The softening and hardening effects were both observed in the microscale.

3. The forced amplitude increases with the increase in excitation amplitude, but the rate of increase is influenced by the detuning parameter and viscoelastic damping parameter. The effects of the nonlocal parameter and viscoelastic damping parameter on the excited vibration of microbeams are coupled. 
Author Contributions: C.L.: Conceptualization, Simulation, Investigation, and Writing-Original draft preparation. C.Z.: Data curation, Software, Methodology, and Validation. S.S.: Formal analysis and Writing-Reviewing and Editing. J.Y.: Resources, Project administration, and Supervision. All authors have read and agreed to the published version of the manuscript.

Funding: This research was supported by the National Natural Science Foundation of China (Nos. 11972240 and 12072112), China Postdoctoral Science Foundation (Nos. 2020M671574 and 2021M700306), and Natural Science Foundation of Jiangxi Province (Grant No. 20202ACBL214014).

Institutional Review Board Statement: Not applicable.

Informed Consent Statement: Not applicable.

Data Availability Statement: Data is contained within the present article. The data presented in this study are available in each figure.

Conflicts of Interest: The authors declare no conflict of interest.

\section{References}

1. Ling, T.; Guo, L.J. Sensitivity enhancement in optical micro-tube resonator sensors via mode coupling. Appl. Phys. Lett. 2013, 103, 013702. [CrossRef]

2. De Pastina, A.; Villanueva, L.G. Suspended micro/nano channel resonators: A review. J. Micromechan. Microengineer. 2020, 30, 043001. [CrossRef]

3. Hu, W.P.; Huai, Y.L.; Xu, M.B.; Feng, X.Q.; Jiang, R.S.; Zheng, Y.P.; Deng, Z.C. Mechanoelectrical flexible hub-beam model of ionic-type solvent-free nanofluids. Mech. Syst. Signal Process. 2021, 159, 107833. [CrossRef]

4. He, L.H.; Lim, C.W.; Wu, B.S. A continuum model for size-dependent deformation of elastic films of nano-scale thickness. Int. J. Solids Struct. 2004, 41, 847-857. [CrossRef]

5. Zhang, W.M.; Yan, H.; Peng, Z.K.; Meng, G. Electrostatic pull-in instability in MEMS/NEMS: A review. Sens. Actuators A Phys. 2014, 214, 187-218. [CrossRef]

6. Lim, C.W.; Li, C.; Yu, J.L. Free torsional vibration of nanotubes based on nonlocal stress theory. J. Sound Vib. 2012, 331, 2798-2808. [CrossRef]

7. Li, L.; Li, X.; Hu, Y. Free vibration analysis of nonlocal strain gradient beams made of functionally graded material. Int. J. Eng. Sci. 2016, 102, 77-92. [CrossRef]

8. Shen, J.P.; Wang, P.Y.; Li, C.; Wang, Y.Y. New observations on transverse dynamics of microtubules based on nonlocal strain gradient theory. Compos. Struct. 2019, 225, 111036. [CrossRef]

9. Chwał, M.; Muc, A. Buckling and Free Vibrations of Nanoplates-Comparison of Nonlocal Strain and Stress Approaches. Appl. Sci. 2019, 9, 1409. [CrossRef]

10. Civalek, Ö.; Uzun, B.; Yayli, M.Ö.; Akgöz, B. Size-dependent transverse and longitudinal vibrations of embedded carbon and silica carbide nanotubes by nonlocal finite element method. Eur. Phys. J. Plus 2020, 135, 101279. [CrossRef]

11. Yao, L.Q.; Ji, C.J.; Shen, J.P.; Li, C. Free vibration and wave propagation of axially moving functionally graded Timoshenko microbeams. J. Braz. Soc. Mech. Sci. Eng. 2020, 42, 137. [CrossRef]

12. Sari, M.S.; Al-Kouz, W.G.; Atieh, A.M. Transverse Vibration of Functionally Graded Tapered Double Nanobeams Resting on Elastic Foundation. Appl. Sci. 2020, 10, 493. [CrossRef]

13. Tang, Y.; Qing, H. Elastic buckling and free vibration analysis of functionally graded Timoshenko beam with nonlocal strain gradient integral model. Appl. Math. Model. 2021, 96, 657-677. [CrossRef]

14. Yan, J.W.; Zhang, W. An atomistic-continuum multiscale approach to determine the exact thickness and bending rigidity of monolayer graphene. J. Sound Vib. 2021, 514, 116464. [CrossRef]

15. Stoimenov, B.L.; Mukai, T. A Model of Adhesion Excited Micro-vibrations. Tribol. Lett. 2008, 29, 129-137. [CrossRef]

16. Yan, J.W.; Zhu, J.H.; Li, C.; Zhao, X.S.; Lim, C.W. Decoupling the effects of material thickness and size scale on the transverse free vibration of BNNTs based on beam models. Mech. Syst. Signal Process. 2022, 166, 108440. [CrossRef]

17. Wang, Y.Z.; Li, F.M. Dynamical properties of nanotubes with nonlocal continuum theory: A review. Sci. China Ser. G Phys. Mech. Astron. 2012, 55, 1210-1224. [CrossRef]

18. Eringen, A.C.; Speziale, C.G.; Kim, B.S. Crack-tip problem in non-local elasticity. J. Mech. Phys. Solids 1977, 25, 339-355. [CrossRef]

19. Eringen, A.C. On differential equations of nonlocal elasticity and solutions of screw dislocation and surface waves. J. Appl. Phys. 1983, 54, 4703-4710. [CrossRef]

20. Yang, X.D.; Lim, C.W. Nonlinear vibrations of nano-beams accounting for nonlocal effect using a multiple scale method. Sci. China Ser. E Technol. Sci. 2009, 52, 617-621. [CrossRef]

21. Heireche, H.; Tounsi, A.; Benzair, A. Scale effect on wave propagation of double-walled carbon nanotubes with initial axial loading. Nanotechnology 2008, 19, 185703. [CrossRef] [PubMed]

22. Lim, C.W. On the truth of nanoscale for nanobeams based on nonlocal elastic stress field theory: Equilibrium, governing equation and static deflection. Appl. Math. Mech. 2010, 31, 37-54. [CrossRef] 
23. Li, C. A nonlocal analytical approach for torsion of cylindrical nanostructures and the existence of higher-order stress and geometric boundaries. Compos. Struct. 2014, 118, 607-621. [CrossRef]

24. Li, C.; Yao, L.Q.; Chen, W.Q.; Li, S. Comments on nonlocal effects in nano-cantilever beams. Int. J. Eng. Sci. 2015, 87, 47-57. [CrossRef]

25. Behera, L.; Chakraverty, S. Recent Researches on Nonlocal Elasticity Theory in the Vibration of Carbon Nanotubes Using Beam Models: A Review. Arch. Comput. Methods Eng. 2017, 24, 481-494. [CrossRef]

26. Liu, C.; Ke, L.L.; Yang, J.; Kitipornchai, S.; Wang, Y.S. Nonlinear vibration of piezoelectric nanoplates using nonlocal Mindlin plate theory. Mech. Adv. Mater. Struct. 2018, 25, 1252-1264. [CrossRef]

27. Arefi, M.; Rabczuk, T. A nonlocal higher order shear deformation theory for electro-elastic analysis of a piezoelectric doubly curved nano shell. Compos. Part B Eng. 2019, 168, 496-510. [CrossRef]

28. Faghidian, S.A. Higher-order nonlocal gradient elasticity: A consistent variational theory. Int. J. Eng. Sci. 2020, $154,103337$. [CrossRef]

29. Hang, Q.; Li, S.F.; Zhang, A.M.; Peng, Y.X. On nonlocal geometrically exact shell theory and modeling fracture in shell structures. Comput. Methods Appl. Mech. Eng. 2021, 386, 114074.

30. Wu, C.P.; Hu, H.X. A review of dynamic analyses of single- and multi-layered graphene sheets/nanoplates using various nonlocal continuum mechanics-based plate theories. Acta Mech. 2021, 232, 4497-4531. [CrossRef]

31. Zhang, N.; Yan, J.W.; Li, C.; Zhou, J. Combined bending-tension/compression deformation of micro-bars accounting for strain-driven long-range interactions. Arch. Mech. 2019, 71, 3-21.

32. Chakravartula, A.; Komvopoulos, K. Viscoelastic properties of polymer surfaces investigated by nanoscale dynamic mechanical analysis. Appl. Phys. Lett. 2006, 88, 131901. [CrossRef]

33. Hettich, M.; Jacob, K.; Ristow, O.; Schubert, M.; Bruchhausen, A.; Gusev, V.; Dekorsy, T. Viscoelastic properties and efficient acoustic damping in confined polymer nano-layers at $\mathrm{GHz}$ frequencies. Sci. Rep. 2016, 6, 33471. [CrossRef] [PubMed]

34. Wu, C.L.; Li, L.D.; Wang, W.S.; Gu, Z.W. Experimental characterization of viscoelastic behaviors of nano-TiO $2 / \mathrm{CaCO}_{3} \mathrm{modified}$ asphalt and asphalt mixture. Nanomaterials 2021, 11, 106. [CrossRef] [PubMed]

35. Rao, S.S. Mechanical Vibrations, 5th ed.; Prentice Hall: Englewood Cliffs, NJ, USA, 2011; pp. 271-281.

36. Ding, H.; Chen, L.Q. Natural frequencies of nonlinear vibration of axially moving beams. Nonlinear Dyn. 2011, 63, 125-134. [CrossRef]

37. Yang, X.D.; Liu, M.; Zhang, W.; Qian, Y.J.; Melnik, R.V.N. On the Perturbation Methods for Vibration Analysis of Linear Time-Varying Systems. Int. J. Appl. Mech. 2016, 8, 1650035. [CrossRef]

38. Nayfeh, A.H.; Mook, D.T. Nonlinear Oscillations; John Wiley \& Sons: New York, NY, USA, 1979; pp. 175-179.

39. Lim, C.W.; Zhang, G.; Reddy, J.N. A higher-order nonlocal elasticity and strain gradient theory and its applications in wave propagation. J. Mech. Phys. Solids 2015, 78, 298-313. [CrossRef] 